PESQUIMAT, Revista de la F.C.M. de la Universidad Nacional Mayor de San Marcos Vol. XV No1, pp. 79-95, Lima - Perú, Marzo 2013

\title{
APLICACIÓN DE FRAMES EN TEOREMAS MUESTRALES
}

Luis Miguel Nuñez Ramirez ${ }^{6}$, Rodolfo Jose Galvez Perez ${ }^{7}$, Humberto Emiliano Galvez Perez ${ }^{8}$

Resumen: Un frame es la generalización del concepto de una base en un espacio vectorial, en este contexto estudiaremos la técnica de los frames, algunas de sus propiedades, y sus aplicaciones en la teoría de muestreo.

Palabras clave: frame, operador frame, cotas frame, teoremas muestrales. Abstract: A frame is the generalization of the concept of a basis in a vector space, in this context we study the technique of the frames, some of its properties, and its applications in sampling theory.

Key words: frame, frame operator, bounded frame, sampling theorems.

\section{Introducción}

El concepto de frames en un espacio de Hilbert fue inicialmente desarrollada por R. Duffin y A. Schaeffer en 1952; cuyo estudio viene relacionado con la teoría de la señal, debido a que los frames analizan la estabilidad y la redundancia de las representaciones lineales de señales discretas. Un frame es una familia de vectores $\left\{g_{n}\right\}_{n \in \mathbb{Z}}$, en un cierto espacio que caracteriza a cualquier señal $f$, vía la familia de sus productos internos $\left\{\left\langle f, g_{n}\right\rangle\right\}_{n \in \mathbb{Z}}$. Los frames proporcionan representaciones estables para las señales, las cuales son de energía finita en $\mathbb{L}^{2}(\mathbb{R})$. En la primera parte de este trabajo se estudia la definición de frames y alguna de sus propiedades, en la segunda parte se estudia la aplicación de los frames a la teoría de muestreo que trata sobre el problema de la recuperación de una función, perteneciente a un cierto espacio funcional, a partir de una sucesión de sus valores o, en general, a partir de una sucesión de valores de ciertas funciones relacionadas con ella.

\section{Definiciones y resultados preliminares}

Sea $\mathbb{H}$ un espacio de Hilbert separable, con producto interno $\langle$,$\rangle y norma$

$$
\|f\|=\sqrt{\langle f, f\rangle} \text {, para todo } f \in \mathbb{H} \text {. }
$$

Definición 2.1. Dada una función $f \in \mathbb{L}^{2}(\mathbb{R})$, definimos los operadores $\mathscr{T}_{a} f, E_{a} f$ y $D_{a} f$ por medio de

$$
\begin{aligned}
\mathscr{T}_{a} f(x) & =f(x-a) \\
E_{a} f(x) & =e^{2 \pi i a x} f(x) \\
D_{a} f(x) & =\frac{1}{\sqrt{|a|}} f\left(\frac{x}{a}\right), a \neq 0, \text { donde } a \text { es un número real. }
\end{aligned}
$$

En particular, $E_{a}(x)=e^{2 \pi i a x}$. Los operadores $\mathscr{T}_{a} f, E_{a} f$ y $D_{a} f$ son operadores unitarios de $\mathbb{L}^{2}(\mathbb{R})$ en $\mathbb{L}^{2}(\mathbb{R})$.

Definición 2.2. La Transformada de Fourier de una función $f$, la definimos por medio de $F: \mathbb{L}^{2}(\mathbb{R}) \longrightarrow \mathbb{L}^{2}(\mathbb{R})$, tal que

$$
F(f)(w)=\widehat{f}(w)=\int_{-\infty}^{\infty} e^{2 \pi i w x} f(x) d x
$$

\footnotetext{
${ }^{6}$ UNMSM, Facultad de Ciencias Matemáticas, Lima- Perú, e-mail: lnuñezr@unmsm.edu.pe

${ }^{7}$ UNMSM, Facultad de Ciencias Matemáticas, Lima - Perú, e-mail: rgalvezp@unmsm.edu.pe

${ }^{8}$ UNMSM, Facultad de Ciencias Matemáticas, Lima - Perú, e- mail: hgalvezp@unmsm.edu.pe
} 
y su inversa viene dada por:

$$
F^{-1}(f)(x)=f(x)=\int_{-\infty}^{\infty} \widehat{f}(x) e^{-2 \pi i w x} d w
$$

\section{Observación 2.3.}

1. Si $f, g \in \mathbb{L}^{2}(\mathbb{R})$, entonces $\langle f, g\rangle_{\mathbb{L}^{2}(\mathbb{R})}=\int_{-\infty}^{\infty} f(x) \overline{g(x)} d x,\|f\|_{2}^{2}=\langle f, f\rangle_{\mathbb{L}^{2}(\mathbb{R})}$.

2. Como $F: \mathbb{L}^{2}(\mathbb{R}) \longrightarrow \mathbb{L}^{2}(\mathbb{R})$ es un operador unitario, se tiene que si $f \in$ $\mathbb{L}^{2}(\dot{\mathbb{R}})$, entonces $\widehat{f} \in \mathbb{L}^{2}(\mathbb{R})$ y $\|f\|_{2}=\|\widehat{f}\|_{2}$.

También, se verifica que:

$$
\begin{aligned}
\left\langle f, \mathscr{T}_{a} g\right\rangle & =\left\langle\mathscr{T}_{-a} f, g\right\rangle \\
\left\langle f, E_{a} g\right\rangle & =\left\langle E_{-a} f, g\right\rangle, \mathrm{y} \\
\left\langle f, D_{a} g\right\rangle & =\left\langle D_{1 / a} f, g\right\rangle, a \neq 0
\end{aligned}
$$

Definicion 2.4. Definimos $S(\mathbb{R})$, como el espacio de funciones de Schwartz, es decir

$$
S(\mathbb{R})=\left\{f \in C^{\infty}(\mathbb{R}): \lim _{|x| \rightarrow \infty} x^{\beta} D^{\alpha} f(x)=0, \forall \alpha, \beta \in \mathbb{Z}^{+}\right\}
$$

Definicion 2.5. Sea $G=\left\{g_{n}\right\}_{n \in \mathbb{Z}}$ una sucesión en $\mathbb{H}$ (no necesariamente es una base), $G$ es un Frame si existen $A, B$ con $0<A \leq B<\infty$, tales que para todo $f \in \mathbb{H}$, se tiene

$$
A\|f\|^{2} \leq \sum_{n \in \mathbb{Z}}\left|\left\langle f, g_{n}\right\rangle\right|^{2} \leq B\|f\|^{2}
$$

Las constantes $A$ y $B$, se denominan cotas frames.

Además, el frame es exacto si al quitar un elemento deja de ser un frame.

Ejemplo: Sea $\mathbb{H}=\mathbb{C}^{2}$ (Complejos), $g_{1}=(1,0), g_{2}=\left(-\frac{1}{2},-\frac{\sqrt{3}}{2}\right)$ y $g_{3}=\left(-\frac{1}{2}, \frac{\sqrt{3}}{2}\right)$. Luego, para todo $z=\left(z_{1}, z_{2}\right) \in \mathbb{C}^{2}$,se tiene

$$
\sum_{i=1}^{3}\left|\left\langle z, g_{i}\right\rangle\right|^{2}=\left|z_{1}\right|^{2}+\left|-\frac{1}{2} z_{1}-\frac{\sqrt{3}}{2} z_{2}\right|^{2}+\left|-\frac{1}{2} z_{1}+\frac{\sqrt{3}}{2} z_{2}\right|^{2}=\frac{3}{2}\|z\|_{\mathbb{C}}^{2}
$$

Por tanto $\left\{g_{1}, g_{2}, g_{3}\right\}$ es un frame con $A=B=\frac{3}{2}$

Definicion 2.6. El conjunto $G=\left\{g_{n}\right\}_{n \in \mathbb{Z}}$ se denomina tight-frame, si $A=B$; luego

$$
\sum_{n \in \mathbb{Z}}\left|\left\langle f, g_{n}\right\rangle\right|^{2}=A\|f\|^{2}
$$

Teorema 2.7. Se tienen los siguientes resultados:

1. Todo frame $G=\left\{g_{n}\right\}$ en $\mathbb{H}$ es completo.

2. Toda base ortonormal en $\mathbb{H}$ es un frame, con cotas frames $A=B=1$.

3. Si $G=\left\{g_{n}\right\}_{n \in \mathbb{Z}}$ es un tight-frame con $A=B=1,\left\|g_{n}\right\|=1$, entonces $\left\{g_{n}\right\}_{n \in \mathbb{Z}}$ es una base ortonormal. 
4. Una base de Shauder de $\mathbb{H}$ no es necesariamente un frame para $\mathbb{H}$.

\section{Demostración.}

1. Previamente recordemos que

$\left\{h_{n}\right\}$ es completa, si $\left\langle f, h_{n}\right\rangle=0$, para cada $n \in \mathbb{N}$ entonces $f=0$

Sea $f \in \mathbb{H}$ arbitrario y $\left\langle f, g_{n}\right\rangle=0$ para cada $n \in \mathbb{N}$, por demostrar que $f=0$.

En efecto, por (1) se tiene

$$
\begin{aligned}
A\|f\|^{2} & \leq \sum_{n \in \mathbb{Z}}\left|\left\langle f, g_{n}\right\rangle\right|^{2}=0 \text { si y solo si } 0 \leq A\|f\|^{2} \leq 0, A>0 \\
\text { entonces }\|f\|^{2} & =0 \text {, luego } f=0 .
\end{aligned}
$$

2. Si $G=\left\{g_{n}\right\}_{n \in \mathbb{Z}}$ es una base ortonormal de $\mathbb{H}$,entonces por la igualdad de Parseval,

$$
\sum_{n \in \mathbb{Z}}\left|\left\langle f, g_{n}\right\rangle\right|^{2}=\|f\|^{2}
$$

entonces $\left\{g_{n}\right\}_{n \in \mathbb{Z}}$ es un tigh-frame con $A=B=1$.

3. Demostrar que $\left\{g_{n}\right\}_{n \in \mathbb{Z}}$ es una base ortonormal de $\mathbb{H}$ es equivalente a probar que $\left\{g_{n}\right\}$ es completo en $\mathbb{H},\left\|g_{n}\right\|=1$ y $\left\langle g_{n}, g_{m}\right\rangle=0, n \neq m$.

En efecto, $\left\{g_{n}\right\}_{n \in \mathbb{Z}}$ es completo, inmediato por la parte (i), $\left\|g_{n}\right\|=1$,para cada $n \in \mathbb{N}$, por hipótesis; y $\left\langle g_{n}, g_{m}\right\rangle=0, n \neq m$, en efecto, por hipótesis, como $\left\{g_{n}\right\}_{n \in \mathbb{Z}}$ es un tigh-frame con $A=B=1$,

$$
\sum_{n \in \mathbb{Z}}\left|\left\langle f, g_{n}\right\rangle\right|^{2}=\|f\|^{2} \text {, para todo } f \in \mathbb{H} \text {. }
$$

Tomemos $f=g_{m}$, entonces

$$
\left\|g_{m}\right\|^{2}=\sum_{n \in \mathbb{Z}}\left|\left\langle g_{m}, g_{n}\right\rangle\right|^{2}=\left|\left\langle g_{m}, g_{m}\right\rangle\right|^{2}+\sum_{n \neq m}\left|\left\langle g_{m}, g_{n}\right\rangle\right|^{2}=\left|\left\|g_{m}\right\|^{2}\right|^{2}+\sum_{n \neq m}\left|\left\langle g_{m}, g_{n}\right\rangle\right|^{2}
$$

entonces $\sum_{n \neq m}\left|\left\langle g_{m}, g_{n}\right\rangle\right|^{2}=0$, luego $0 \leq\left|\left\langle g_{m}, g_{n}\right\rangle\right|^{2} \leq \sum_{n \neq m}\left|\left\langle g_{m}, g_{n}\right\rangle\right|^{2} \leq 0$

por lo que se deduce que $\left|\left\langle g_{m}, g_{n}\right\rangle\right|^{2}=0$, por lo tanto $\left\langle g_{m}, g_{n}\right\rangle=0, n \neq m$.

4. Previamente, recordemos que $\left\{x_{n}\right\}$ en $\mathbb{H}$ es llamada una base de Shauder, si existe una única $\left\{c_{n}\right\}$ tal que

$$
x=\sum_{n \in \mathbb{Z}} c_{n} x_{n}, \text { para todo } x \in \mathbb{H}
$$

En efecto, sea $\widetilde{G}=\left\{\widetilde{g}_{n}\right\}_{n \in \mathbb{Z}}$ una base ortonormal (debido a que todo espacio de Hilbert posee una base ortonormal). Definamos $G=\left\{g_{n}\right\}$, mediante

$$
g_{n}=n \widetilde{g}_{n}
$$

entonces como $\left\{\widetilde{g}_{n}\right\}$ es una base ortonormal esto implica que $\left\{g_{n}\right\}_{n \in \mathbb{Z}}$ es linealmente independiente y completa, esto es, $\left\{g_{n}\right\}$ es una base de Shauder . 
Sin embargo, tomemos $m$ arbitrario

$$
\begin{aligned}
\sum_{n \in \mathbb{Z}}\left|\left\langle g_{m}, g_{n}\right\rangle\right|^{2} & =\sum_{n \in \mathbb{Z}}\left|\left\langle m \widetilde{g}_{m}, n \widetilde{g}_{n}\right\rangle\right|^{2}=\sum_{n \in \mathbb{Z}}\left|m\left\langle\widetilde{g}_{m}, n \widetilde{g}_{n}\right\rangle\right|^{2} \\
& =m^{2} \sum_{n \in \mathbb{Z}}\left|\left\langle\widetilde{g}_{m}, \widetilde{g}_{n}\right\rangle\right| n^{2} \\
& =m^{2} m^{2}=m^{4}
\end{aligned}
$$

(esto pues, como $\left\{\widetilde{g}_{n}\right\}_{n \in \mathbb{Z}}$ es una base ortonormal, entonces $\left\langle\widetilde{g}_{m}, \widetilde{g}_{n}\right\rangle=0$,si $n \neq m$ ) esto es,

$$
\sum_{n \in \mathbb{Z}}\left|\left\langle g_{m}, g_{n}\right\rangle\right|^{2}=m^{4}, m \text { arbitrario . }
$$

Pero $\sum_{n \in \mathbb{Z}}\left|\left\langle g_{m}, g_{n}\right\rangle\right|^{2}=m^{4} \underset{m \longrightarrow \infty}{\longrightarrow}+\infty$, entonces

$$
\text { no existe } B<\infty \text { tal que } \sum_{n \in \mathbb{Z}}\left|\left\langle g_{m}, g_{n}\right\rangle\right|^{2} \leq B\left\|g_{m}\right\|^{2} \leq B
$$

por lo tanto $\left\{g_{n}\right\}$ no constituye un frame.

Definición 2.8. Sea $\mathbb{H}$ un espacio de Hilbert Separable, y $G=\left\{g_{n}\right\}_{n \in \mathbb{Z}} \subset \mathbb{H}$ un frame con cotas frames $A$ y $B$, definamos $S: \mathbb{H} \longrightarrow \mathbb{H}$, tal que $S f=: \sum_{n \in \mathbb{Z}}\left\langle f, g_{n}\right\rangle g_{n}$, su serie frame, $S$ es llamado un operador frame.

\section{Observación 2.9.}

1. El operador frame $S$ es un operador lineal y acotado sobre $\mathbb{H}$ con $A I \leq S \leq B I$.

2. $S$ es inversible con $B^{-1} I \leq S^{-1} \leq A^{-1} I$.Además $S^{-1}$ es un operador positivo $\left(S^{-1} \geq 0\right)$, entonces $S^{-1}$ es auto-adjunto .

3. $\left\{S^{-1} g_{n}\right\}$ es un frame con cotas frames $B^{-1}$ y $A^{-1}$.

4. Para cada $f \in \mathbb{H}, f=\sum_{n \in \mathbb{Z}}\left\langle f, S^{-1} g_{n}\right\rangle g_{n}=\sum_{n \in \mathbb{Z}}\left\langle f, g_{n}\right\rangle S^{-1} g_{n}$.

5. Si existe $\left\{c_{n}\right\}$, tal que $f=\sum_{n \in \mathbb{Z}} c_{n} g_{n}$, entonces $\sum_{n \in \mathbb{Z}}\left|c_{n}\right|^{2}=\sum_{n \in \mathbb{Z}}\left|a_{n}\right|^{2}+\sum_{n \in \mathbb{Z}}\left|a_{n}-c_{n}\right|^{2}$, esto es $\sum_{n \in \mathbb{Z}}\left|a_{n}\right|^{2} \leq \sum_{n \in \mathbb{Z}}\left|c_{n}\right|^{2}$, donde $a=\left\langle f, S^{-1} g_{n}\right\rangle$.

6. Si adicionalmente, $\left\{g_{n}\right\}_{n \in \mathbb{Z}}$ es un frame exacto, entonces $\left\{g_{n}\right\}$ y $\left\{S^{-1} g_{n}\right\}$ son biortogonales, esto es

$$
\left\langle g_{m}, S^{-1} g_{n}\right\rangle=\delta_{m, n}=\left\{\begin{array}{l}
1, m=n \\
0, m \neq n
\end{array}\right.
$$

Teorema 2.10. Sea $g \in \mathbb{L}^{2}(\mathbb{R})$ tal que $G(x)=\sum_{n \in \mathbb{Z}}|g(x-n a)|^{2}$ satisface $0<A \leq G(x) \leq$ $B<\infty$, en casi todo punto sobre $\mathbb{R}$. Si g tiene soporte compacto, con soport $(g) \subset I$, donde $I$ es un intervalo de longitud $\frac{1}{b}$, entonces $\left\{E_{m b} T_{n a} g\right\}_{m, n \in \mathbb{Z}}$ es un frame con cotas frames $\frac{A}{b}$ y $\frac{B}{b}$. Además

$$
\text { para todo } f \in \mathbb{L}^{2}(\mathbb{R}), S f=\frac{1}{b} G(x) f(x)
$$

donde $S$ es el operador frame; asimismo, $S$ es invertible y

$$
S^{-1} f(x)=\frac{b f(x)}{G(x)}
$$




\section{Demostración.}

En primer lugar demostraremos la relación (3).

En efecto, como $S^{-1} f \in \mathbb{L}^{2}(\mathbb{R})$, tomemos $S^{-1} f$ por $f$ en (2) y obtenemos

$$
S\left(S^{-1} f\right)=\frac{1}{b} G(x) S^{-1} f(x),
$$

entonces $f(x)=\frac{G(x)}{b} S^{-1} f(x)$, esto es $S^{-1} f(x)=\frac{b f(x)}{G(x)}$.

Luego, probaremos que $\left\{E_{m b} T_{n a} g\right\}_{m, n \in \mathbb{Z}}$ es un frame para $\mathbb{L}^{2}(\mathbb{R})$ con cotas frames $A b^{-1}$ y $B b^{-1}$ y la relación (2). En efecto, Si soport $(g) \subset I$ entonces $\operatorname{soport}\left(\mathscr{T}_{\text {na }} g\right) \subset I_{n}$, donde

$$
I_{n}=\{x+n a: x \in I\}, \mathrm{y}, \mathscr{T}_{n a} g(x)=g(x-n a) .
$$

Fijemos $n$, consideremos $h_{n}(x)=f(x) \overline{g(x-n a)} \in \mathbb{L}^{2}\left(I_{n}\right)$ (en efecto, como $f \in \mathbb{L}^{2}(\mathbb{R}), \mathscr{T}_{n a} g \in$ $\mathbb{L}^{2}(\mathbb{R})$ con $\mathscr{T}_{n a} g$ con soporte compacto entonces $\left.f \mathscr{T}_{n a} g \in \mathbb{L}^{2}\left(I_{n}\right)\right)$.

Pero también $e_{m}(x)=\sqrt{b} E_{m b}(x)=b^{1 / 2} e^{2 \pi i m b x}$ es una base ortonormal de $\mathbb{L}^{2}\left(I_{n}\right)$. Por lo tanto $h_{n}(x)=\sum_{m \in \mathbb{Z}}\left\langle h_{n}, e_{m}\right\rangle e_{m}$, y por la igualdad de Parseval,

$$
\left\|h_{n}\right\|_{\mathbb{L}^{2}}^{2}=\int_{I_{n}}\left|h_{n}(x)\right|^{2} d x=\sum_{m \in \mathbb{Z}}\left|\left\langle h_{n}, e_{m}\right\rangle\right|^{2}
$$

Asimismo

$$
\begin{array}{rl}
\int_{I_{n}}\left|h_{n}(x)\right|^{2} & d x=\sum_{m \in \mathbb{Z}}\left|b \int_{I_{n}} f(x) \overline{g(x-n a)} E_{m b}(x) d x\right|^{2} \\
& =b \sum_{m \in \mathbb{Z}}\left|\int_{I_{n}} f(x) E_{m b} T_{n a} g(x) d x\right|^{2} \\
& =b \sum_{m \in \mathbb{Z}}\left|\left\langle f, E_{m b} T_{n a} g\right\rangle\right|^{2}
\end{array}
$$

entonces

$$
\begin{aligned}
\sum_{n \in \mathbb{Z}} \int_{I_{n}}\left|h_{n}(x)\right|^{2} d x= & b \sum_{n \in \mathbb{Z}} \sum_{m \in \mathbb{Z}}\left|\left\langle f, E_{m b} T_{n a} g\right\rangle\right|^{2} \\
= & \sum_{n \in \mathbb{Z}} \int_{I_{n}}|f(x)|^{2}|g(x-n a)|^{2} d x=\sum_{n \in \mathbb{Z}} \int_{-\infty}^{\infty}|f(x)|^{2}|g(x-n a)|^{2} d x \\
& (g \text { de soporte compacto }) \\
= & \int|f(x)|^{2} \sum_{n \in \mathbb{Z}}|g(x-n a)|^{2} d x=\int|f(x)|^{2} G(x) d x \\
\text { donde } G(x)= & \sum_{n \in \mathbb{Z}}|g(x-n a)|^{2}
\end{aligned}
$$

Por consiguiente

$$
\begin{aligned}
b \sum_{n, m \in \mathbb{Z}}\left|\left\langle f, E_{m b} T_{n a} g\right\rangle\right|^{2} & =\int|f(x)|^{2} G(x) d x \text { esto es, } \\
\sum_{n, m \in \mathbb{Z}}\left|\left\langle f, E_{m b} T_{n a} g\right\rangle\right|^{2} & =\frac{1}{b} \int|f(x)|^{2} G(x) d x
\end{aligned}
$$


Entonces por teorema 2.7, se tiene

$$
\frac{A}{b}\|f\|^{2} \leq \sum_{n, m \in \mathbb{Z}}\left|\left\langle f, E_{m b} T_{n a} g\right\rangle\right|^{2} \leq \frac{B}{b} \int|f(x)|^{2} d x=\frac{B}{b}\|f\|^{2}
$$

por lo tanto $\left\{E_{m b} T_{n a} g\right\}_{m, n \in \mathbb{Z}}$ es un frame para $\mathbb{L}^{2}(\mathbb{R})$ con cotas frames $\frac{A}{b}, \frac{B}{b}$.

Afirmación: $(S f)(x)=\frac{1}{b} f(x) G(x) S$ es un operador frame.

En efecto, por definición de $S$, y como $\left\{E_{m b} T_{n a} g\right\}$ es un frame, se tiene

$$
\begin{aligned}
S f(x) & =\sum_{m, n \in \mathbb{Z}}\left\langle f, E_{m b} T_{n a} g\right\rangle E_{m b} T_{n a} g, \operatorname{como} E_{m b} T_{n a} g(x)=e^{2 \pi i m b x} g(x-n a) \\
& =\sum_{n \in \mathbb{Z}}\left[\sum_{m \in \mathbb{Z}}\left\langle f, E_{m b} T_{n a} g\right\rangle e^{2 \pi i m b x}\right] g(x-n a) \\
& =\sum_{n \in \mathbb{Z}} H_{n}(x) g(x-n a), H_{n}(x)=\sum_{m \in \mathbb{Z}}\left\langle f, E_{m b} T_{n a} g\right\rangle e^{2 \pi i m b x}
\end{aligned}
$$

A su vez, $H_{n}$ es periódica de periódo $\frac{1}{b}$. En efecto

$$
\begin{aligned}
H_{n}\left(x+\frac{1}{b}\right) & =\sum_{m \in \mathbb{Z}}\left\langle f, e^{2 \pi i m b\left(x+\frac{1}{b}\right)} g\left(x+\frac{1}{b}-n a\right)\right\rangle e^{2 \pi i m b\left(x+\frac{1}{b}\right)} \\
e^{2 \pi i m b\left(x+\frac{1}{b}\right)} & =e^{2 \pi i m b x} e^{2 \pi i m}=e^{2 \pi i m b x} \\
g\left(x+\frac{1}{b}-n a\right) & =g\left(x-\left(n a-\frac{1}{b}\right)\right)
\end{aligned}
$$

Por consiguiente, $H_{n}\left(x+\frac{1}{b}\right)=H_{n}(x)$.

Por lo tanto, por análisis de Fourier, se tiene

$$
\int_{I_{n}} H_{n}(x) e^{-2 \pi i m b x} d x=\frac{1}{b} \int_{I_{n}} f(x) \overline{g(x-n a)} e^{-2 \pi i m b x} d x
$$

En efecto

$$
\begin{aligned}
\int_{I_{n}} H_{n}(x) e^{-2 \pi i m b x} d x & =\left\langle H_{n}(x), e^{2 \pi i m b x}\right\rangle_{\mathbb{L}^{2}\left(I_{n}\right)}=\left\langle\sum_{m \in \mathbb{Z}}\left\langle f, E_{m b} T_{n a} g\right\rangle e^{2 \pi i m b x}, e^{2 \pi i k b x}\right\rangle \\
& =\sum_{m \in \mathbb{Z}}\left\langle f, E_{m b} T_{n a} g\right\rangle\left\langle e^{2 \pi i m b x}, e^{2 \pi i k b x}\right\rangle \\
& =\left\langle f, E_{m b} T_{n a} g\right\rangle_{\mathbb{L}^{2}\left(I_{n}\right)}=\frac{1}{b} \int_{I_{n}} f(x) \overline{g(x-n a)} e^{-2 \pi i m b x} d x
\end{aligned}
$$

Entonces

$$
\begin{aligned}
\int_{I_{n}}\left[H_{n}(x)-\frac{1}{b} f(x) \overline{g(x-n a)}\right] e^{-2 \pi i m b x} d x & =0 \\
\left\langle\left(H_{n}(x)-\frac{1}{b} f(x) \overline{g(x-n a)}\right), e^{-2 \pi i m b x}\right\rangle_{\mathbb{L}^{2}\left(I_{n}\right)} & =0 \text {, para todo }\left\{e^{-2 \pi i m b x}\right\}_{m \in \mathbb{Z}}
\end{aligned}
$$

y como $\left\{e^{-2 \pi i m b x}\right\}_{m \in \mathbb{Z}}$ es completa (debido a que $\left\{e^{-2 \pi i m b x}\right\}_{m \in \mathbb{Z}}$ es una base ortonormal, entonces $\left\{e^{-2 \pi i m b x}\right\}_{m \in \mathbb{Z}}$ es un frame) 
Por lo tanto

$$
\left[H_{n}(x)-\frac{1}{b} f(x) \overline{g(x-n a)}\right]=0, \text { esto es, } H_{n}(x)=\frac{1}{b} f(x) \overline{g(x-n a)}
$$

Luego

$$
\begin{aligned}
S f(x) & =\sum_{n \in \mathbb{Z}} H_{n}(x) g(x-n a)=\sum_{n \in \mathbb{Z}} \frac{1}{b} f(x) \overline{g(x-n a)} g(x-n a) \\
& =\frac{1}{b} \sum_{n \in \mathbb{Z}} f(x)|g(x-n a)|^{2}=\frac{1}{b} f(x) \sum_{n \in \mathbb{Z}}|g(x-n a)|^{2}
\end{aligned}
$$

Por lo tanto

$$
S f(x)=\frac{1}{b} f(x) G(x)
$$

\section{Teoremas Muestrales}

\section{Teorema 3.1}

Sea $\Omega, \sigma$ reales positivos, tales que $0<\Omega<\frac{1}{2 \sigma}$, y sea $g \in S(\mathbb{R})$, tal que soport $(\widehat{g}) \subset$ $\left[-\frac{1}{2 \Omega}, \frac{1}{2 \Omega}\right] ; \hat{g}(w)=1$ para $|w| \leq \sigma$ y $\widehat{g}>0$ sobre $\left(-\frac{1}{2 \Omega},-\sigma\right] \cup\left[\sigma, \frac{1}{2 \Omega}\right)$.

Sea $G(w)=\sum_{m \in \mathbb{Z}}|\widehat{g}(w-m b)|^{2}$ y definamos $\phi$ via $\widehat{\phi}(w)=\frac{\widehat{g}(w)}{G(w)}$, donde $\sigma+\frac{1}{2 \Omega} \leq b<\frac{1}{\Omega}$.

Entonces para toda función $f \sigma$-banda limitada, se tiene

$$
f(t)=\Omega \sum_{n \in \mathbb{Z}} f(n \Omega) \phi(t-n \Omega) \text { en } \mathbb{L}^{2}(\mathbb{R})
$$

\section{Demostración.}

En efecto, sabemos que $g \in S(\mathbb{R})$ entonces $\hat{g} \in S(\mathbb{R})$ y $\operatorname{soport}(\widehat{g})$ es compacto. También, $\operatorname{soport}(\widehat{f}) \subset I=[-\sigma, \sigma],|I|<\frac{1}{\Omega}$.

Además, puesto que $\operatorname{soport}(\widehat{g})$ es compacto y soport $(\widehat{g})$ está contenido en un intervalo de longitud $\frac{1}{\Omega}$, esto es $\operatorname{soport}(\widehat{g}) \subset\left[-\frac{1}{2 \Omega}, \frac{1}{2 \Omega}\right]$, con $\left|\left[-\frac{1}{2 \Omega}, \frac{1}{2 \Omega}\right]\right|=\frac{1}{2 \Omega}-\left(-\frac{1}{2 \Omega}\right)=\frac{1}{\Omega}$, para $m$ suficientemente grande $\widehat{g}(w-m b)$ se anula, entonces existen $A, B$, tal que

$$
0<A \leq G(w)=\sum_{m \in \mathbb{Z}}|\widehat{g}(w-m b)|^{2} \leq B<\infty, \text { en casi todo punto. }
$$

Nuevamente aplicando el teorema 2.10, tenemos $\left\{E_{n a} T_{m b} \widehat{g}\right\}$ es un frame para $\mathbb{L}^{2}(\mathbb{R})$, donde $a=\Omega$.

Por lo tanto

$$
\begin{aligned}
\widehat{f} & =\sum_{m, n \in \mathbb{Z}}\left\langle\widehat{f}, E_{n a} T_{m b} \widehat{g}\right\rangle S^{-1} E_{n a} T_{m b} \widehat{g} \\
& =\sum_{m, n \in \mathbb{Z}}\left\langle\widehat{f}, E_{n a} T_{m b} \widehat{g}\right\rangle E_{n a} T_{m b}\left(S^{-1} \widehat{g}\right)
\end{aligned}
$$

Afirmación: $S^{-1}(\widehat{g})=\Omega \frac{\widehat{g}}{G}$. 
En efecto, de acuerdo al teorema 2.10, $S^{-1} f(x)=b \frac{f(x)}{G(x)}$, tomando $f=\widehat{g}, b=\Omega$, entonces

$$
S^{-1}(\widehat{g}(x))=\Omega \frac{\widehat{g}(x)}{G(x)}=\Omega \widehat{\phi}(x)
$$

esto es,

Pero,

$$
\begin{aligned}
& \widehat{f}=\Omega \sum_{m, n \in \mathbb{Z}}\left\langle\widehat{f}, E_{n a} T_{m b} \widehat{g}\right\rangle E_{n a} T_{m b} \widehat{\phi} \\
& \widehat{f}=\Omega \sum_{m, n \in \mathbb{Z}}\left\langle\widehat{f}, E_{n \Omega} T_{m b} \widehat{g}\right\rangle E_{n \Omega} T_{m b} \widehat{\phi}
\end{aligned}
$$

$$
\left\langle\widehat{f}, E_{n \Omega} T_{m b} \widehat{g}\right\rangle=\int \widehat{f}(w) e^{-2 \pi i n \Omega w} \overline{\widehat{g}(w-m b)} d w
$$

Desde que $\widehat{g}$ tiene soporte en $\left[-\frac{1}{2 \Omega}, \frac{1}{2 \Omega}\right]$, para $m \neq 0$ suficientemente grande,

$$
\widehat{g}(w-m b)=0 \text {; excepto cuando } m=0, \widehat{g}(w)=1 \text { para }|w| \leq \sigma
$$

entonces

$$
\left\langle\widehat{f}, E_{n \Omega} T_{m b} \widehat{g}\right\rangle=\int_{-\infty}^{\infty} \widehat{f}(w) e^{-2 \pi i n \Omega w} \overline{\widehat{g}(w)} d w=\int_{-\sigma}^{\sigma} \widehat{f}(w) e^{-2 \pi i n \Omega w} d w=f(n \Omega)
$$

esto es,

$$
\left\langle\widehat{f}, E_{n \Omega} T_{m b} \widehat{g}\right\rangle=\left\langle\widehat{f}, E_{n \Omega} \widehat{g}\right\rangle=f(n \Omega)
$$

Por lo tanto de (5) y (6), obtenemos

$$
\widehat{f}=\Omega \sum_{n \in \mathbb{Z}} f(n \Omega) E_{n \Omega} T_{0 b} \widehat{\phi}=\Omega \sum_{n \in \mathbb{Z}} f(n \Omega) E_{n \Omega} \widehat{\phi}
$$

Finalmente, usaremos que

$$
\left(E_{b_{m}} T_{a_{n}} \widehat{g}\right)^{\vee}=e^{2 \pi i b_{m} a_{n}} E_{-a_{n}} T_{b_{m}} g
$$

En particular, $b_{m}=n \Omega, a_{n}=m b, g \equiv \phi$

Entonces

$$
\left(E_{n \Omega} T_{m b} \widehat{\phi}\right)^{\vee}=e^{2 \pi i n \Omega(m b)} E_{-m b} T_{n \Omega} \phi
$$

para $m=0$,

$$
\left(E_{n \Omega} T_{0 b} \widehat{\phi}\right)^{\vee}=\left(E_{n \Omega} \widehat{\phi}\right)^{\vee}=E_{-0 b} T_{n \Omega} \phi=T_{n \Omega} \phi
$$

esto es,

$$
E_{n \Omega} \widehat{\phi}=\left(T_{n \Omega} \phi\right)^{\wedge}
$$

Por consiguiente

$$
\widehat{f}=\Omega \sum_{n \in \mathbb{Z}} f(n \Omega)\left(T_{n \Omega} \phi\right)^{\wedge}
$$

y aplicando la anti-transformada de Fourier, obtenemos

$$
f=\Omega \sum_{n \in \mathbb{Z}} f(n \Omega) T_{n \Omega} \phi=\Omega \sum_{n \in \mathbb{Z}} f(n \Omega) \phi(t-n \Omega) \quad \text { en } \mathbb{L}^{2}(\mathbb{R})
$$


Para obtener un teorema de muestreo para muestras irregulares pero usando frames, necesitamos adicionalmente propiedades de frames que estableceremos en el siguiente teorema. Teorema 3.2. Sea $g$ una funcion $\sigma$ - banda limitada y asumamos que $\left\{a_{n}\right\},\left\{b_{m}\right\}$ son números reales por la cual $\left\{E_{a_{n}}(x)\right\}$ es un frame para $\mathbb{L}^{2}([-\sigma, \sigma])$. Asumamos que existan $A, B$ positivos, tal que $0<A \leq G(w)=\sum_{m \in \mathbb{Z}}\left|\widehat{g}\left(w-b_{m}\right)\right|^{2} \leq B<\infty$. Entonces $\left\{E_{a_{n}} T_{b_{m}} \widehat{g}\right\}$, es un frame para $\mathbb{L}^{2}(\mathbb{R})$.

\section{Demostración.}

Sea $I=[-\sigma, \sigma]$ y $I_{m}=I+b_{m}=\left\{x \in b_{m}: x \in I\right\}$.

Afirmación: Para $m$ fijo, $\left\{T_{b_{m}} E_{a_{n}}(x)\right\}$ es un frame para $I_{m}$ con las mismas cotas frames que $\operatorname{para}\left\{E_{a_{n}}(x)\right\}$. En efecto,

$$
\begin{aligned}
T_{b_{m}} E_{a_{n}}(x) & =T_{b_{m}}\left(e^{2 \pi i a_{n} x}\right)=e^{2 \pi i a_{n}\left(x-b_{m}\right)} \\
& =e^{2 \pi i a_{n} x} e^{-2 \pi i a_{n} b_{m}}=e^{2 \pi i a_{n} x}
\end{aligned}
$$

esto es

$$
T_{b_{m}} E_{a_{n}}(x)=e^{2 \pi i a_{n} x}=E_{a_{n}}(x),
$$

$m$ fijado es un frame para $\mathbb{L}^{2}\left(I_{m}\right)$ con las mismas cotas frames que para $\left\{E_{a_{n}}(x)\right\}$, digamos $A_{I}$ y $B_{I}$.

Afirmación: $\widehat{f} T_{b_{m}} \overline{\hat{g}} \in \mathbb{L}^{2}\left(I_{m}\right)$, para todo $f \in \mathbb{L}^{2}(\mathbb{R})$.

En efecto

$$
\begin{aligned}
\int_{I_{m}}\left|\widehat{f} T_{b_{m}} \overline{\hat{g}}\right|^{2} d w & =\int_{I_{m}}|\widehat{f}|^{2}\left|T_{b_{m}} \overline{\hat{g}}\right|^{2} d w \\
& =\int_{I_{m}}|\widehat{f}(w)|^{2}\left|\overline{\hat{g}}\left(w-b_{m}\right)\right|^{2} d w \\
& \leq \sum_{m \in \mathbb{Z}} \int_{I_{m}}|\widehat{f}(w)|^{2}\left|\widehat{g}\left(w-b_{m}\right)\right|^{2} d w \\
& \leq \int_{I_{m}}|\widehat{f}(w)|^{2}\left(\sum_{m \in \mathbb{Z}}\left|\widehat{g}\left(w-b_{m}\right)\right|^{2}\right) d w \\
& \leq \int_{I_{m}}|\widehat{f}(w)|^{2} G(w) d w \leq B \int_{I_{m}}|\widehat{f}(w)|^{2} d w \\
(\text { debido a } 0 & <A \leq G(w) \leq B<\infty) \\
& \leq B \int_{\mathbb{R}}|\widehat{f}(w)|^{2} d w \leq B\|\widehat{f}\|_{\mathbb{L}^{2}(\mathbb{R})}=B\|f\|_{\mathbb{L}^{2}(\mathbb{R})}<\infty
\end{aligned}
$$

Por lo tanto, $\widehat{h}_{m}=\widehat{f} T_{b_{m}} \overline{\widehat{g}} \in \mathbb{L}^{2}\left(I_{m}\right), \forall f \in \mathbb{L}^{2}(\mathbb{R})$.

Afirmación: soport $\left(\widehat{h}_{m}\right) \subset I_{m}$, donde $I_{m}=I+b_{m}$.

En efecto,

$$
\begin{aligned}
\operatorname{soport}\left(\widehat{h}_{m}\right) & =\operatorname{soport}\left(\widehat{f} T_{b_{m}} \overline{\hat{g}}\right) \subseteq \operatorname{soport}(\hat{f}) \cap \operatorname{soport}\left(T_{b_{m}} \overline{\hat{g}}\right) \\
& \subseteq \operatorname{soport}\left(T_{b_{m}} \overline{\hat{g}}\right)=\operatorname{soport}(\overline{\hat{g}}(w-b m)) \\
& \subseteq b_{m}+\operatorname{soport}(\overline{\hat{g}}) \subseteq b_{m}+\overline{[-\sigma, \sigma]}
\end{aligned}
$$

esto es,

$$
\operatorname{soport}\left(\widehat{h}_{m}\right) \subseteq b_{m}+\overline{[-\sigma, \sigma]}=I_{m}
$$


Por lo tanto, $\widehat{h}_{m}$ tiene soporte compacto y soport $\left(\widehat{h}_{m}\right) \subseteq I_{m}$.

Además, como $\left\{T_{b_{m}} E_{a_{n}}\right\}$ es un frame para $I_{m}$ con cotas frames $A_{I}, B_{I}$, entonces,

$$
A_{I}\left\|\widehat{h}_{m}\right\|_{I_{m}}^{2} \leq \sum_{n \in \mathbb{Z}}\left|\left\langle\widehat{h}_{m}, T_{b_{m}} E_{a_{n}}\right\rangle_{I_{m}}\right|^{2} \leq B_{I}\left\|\widehat{h}_{m}\right\|_{I_{m}},
$$

y aplicando sumatoria bajo $m$, obtenemos

$$
A_{I} \sum_{m \in \mathbb{Z}}\left\|\widehat{f} T_{b_{m}} \overline{\widehat{g}}\right\|_{I_{m}}^{2} \leq \sum_{m, n \in \mathbb{Z}}\left|\left\langle\widehat{f} T_{b_{m}} \overline{\widehat{g}}, T_{b_{m}} E_{a_{n}}\right\rangle_{I_{m}}\right|^{2} \leq B_{I} \sum_{m \in \mathbb{Z}}\left\|\widehat{f} T_{b_{m}} \overline{\widehat{g}}\right\|_{I_{m}}^{2}
$$

Asimismo

$$
\begin{aligned}
& \sum_{m \in \mathbb{Z}}\left\|\widehat{f} T_{b_{m}} \overline{\widehat{g}}\right\|_{I_{m}}^{2}=\sum_{m \in \mathbb{Z}} \int_{I_{m}}\left|\widehat{f} \overline{T_{b_{m}}} \overline{\hat{g}}\right|^{2} d w=\sum_{m \in \mathbb{Z}} \int_{I_{m}}|\widehat{f}(w)|^{2}\left|\widehat{g}\left(w-b_{m}\right)\right|^{2} d w \\
& =\int_{-\infty}^{+\infty}|\widehat{f}(w)|^{2}\left(\sum_{m \in \mathbb{Z}}\left|\widehat{g}\left(w-b_{m}\right)\right|^{2}\right) d w=\int_{-\infty}^{+\infty}|\widehat{f}(w)|^{2} G(w) d w
\end{aligned}
$$

debido a que $\widehat{g}$ es de soporte compacto y $G(w)=\sum_{m \in \mathbb{Z}}\left|\widehat{g}\left(w-b_{m}\right)\right|^{2}$.

Afirmación: $\left\langle\widehat{f} T_{b_{m}} \overline{\widehat{g}}, T_{b_{m}} E_{a_{n}}\right\rangle_{I_{m}}=\left\langle\widehat{f}, T b_{m}\left(E_{a_{n}} \widehat{g}\right)\right\rangle_{I_{m}}$

$$
=e^{2 \pi i a_{n} b_{m}} \int_{-\infty}^{+\infty} \widehat{f}(w) e^{-2 \pi i a_{n} w} \overline{\widehat{g}\left(w-b_{m}\right)} d w=e^{2 \pi i a_{n} b_{m}}\left\langle\widehat{f}, E_{a_{n}} T_{b_{m}} \widehat{g}\right\rangle
$$

en efecto

$$
\begin{aligned}
\left\langle\widehat{f} T_{b_{m}} \overline{\widehat{g}}, T_{b_{m}} E_{a_{n}}\right\rangle_{I_{m}} & =\int_{I_{m}}\left(\widehat{f} T_{b_{m}} \overline{\widehat{g}}\right)\left(\overline{T_{b_{m}} E_{a_{n}}}\right) \\
& =\int_{I_{m}}(\widehat{f}(w))\left(\overline{\widehat{g}}\left(w-b_{m}\right)\right) \overline{T_{b_{m}}\left(e^{2 \pi i a_{n} w}\right)} \\
& =\int_{I_{m}} \widehat{f}(w) \overline{\widehat{g}}\left(w-b_{m}\right) e^{-2 \pi i a_{n}\left(w-b_{m}\right)} \\
& =\int_{I_{m}} \widehat{f}(w) \overline{\widehat{g}}\left(w-b_{m}\right) e^{-2 \pi i a_{n} w} e^{2 \pi i a_{n} b_{m}} d w \\
& =e^{2 \pi i a_{n} b_{m}} \int_{-\infty}^{+\infty} \widehat{f}(w) \overline{\widehat{g}\left(w-b_{m}\right) e^{2 \pi i a_{n} w}} d w \\
& =e^{2 \pi i a_{n} b_{m}}\left\langle\widehat{f}, E_{a_{n}} T_{b_{m}} \widehat{g}\right\rangle
\end{aligned}
$$

puesto que $\operatorname{soport}(\widehat{g})=[-\sigma, \sigma]$. Finalmente, de (7), se tiene que

$$
A\|f\|^{2} \leq \sum_{m \in \mathbb{Z}}\left\|\widehat{f} T_{b_{m}} \overline{\hat{g}}\right\|_{I_{m}}^{2}=\int_{-\infty}^{+\infty}|\widehat{f}(w)|^{2} G(w) d w \leq B \int_{-\infty}^{+\infty}|\widehat{f}(w)|^{2} d w=B\|f\|^{2}
$$

Por lo tanto de $(7),(8)$ y $(9)$ en $(7)$, obtenemos

$$
A_{I} A\|f\|^{2} \leq \sum_{m, n \in \mathbb{Z}}\left|\left\langle\widehat{f}, E_{a_{n}} T_{b_{m}} \hat{g}\right\rangle\right|^{2} \leq B_{I} B\|f\|^{2}
$$

De este modo, $\left\{E_{a_{n}} T_{b_{m}} \widehat{g}\right\}$ es un frame para $\mathbb{L}^{2}(\mathbb{R})$. 


\section{Observación 3.3.}

1. Se tiene que $\left\{E_{a_{n}} T_{b_{m}} \widehat{g}\right\}$ es un tight-frame si, y sólo si $\left\{E_{a_{n}}(x)\right\}$ es un tigh-frame para $\mathbb{L}^{2}([-\sigma, \sigma])$ y $G$ es una constante en casi todo punto sobre $\mathbb{R}$. Veamos, de (??), se tiene $A_{I} A=B_{I} B$ esto es, $A_{I}=B_{I}, A=B$.

Por consiguiente, $\left\{E_{a_{n}}(x)\right\}$ es un tigh-frame para $\mathbb{L}^{2}([-\sigma, \sigma])$, y

$$
0<B=A \leq G(w) \leq B<\infty, \text { esto es, } G(w)=A=B
$$

en casi todo punto sobre $\mathbb{R}$.

2. Ademas, el operador frame para $\left\{E_{a_{n}} T_{b_{m}} \widehat{g}\right\}$ es dado mediante

$$
S \widehat{h}=\sum_{m \in \mathbb{Z}} T_{b_{m}} \widehat{g} S_{m}\left(\widehat{h} T_{b_{m}} \overline{\hat{g}}\right)
$$

donde $S_{m}$ es el operador frame para $\left\{T_{b_{m}} E_{a_{n}}\right\}$ que es dado por

$$
S_{m} \widehat{k}=\sum_{n \in \mathbb{Z}}\left\langle\widehat{k}, T_{b_{m}} E_{a_{n}}\right\rangle_{I_{m}} T_{b_{m}} E_{a_{n}}
$$

para $\widehat{h} \in \mathbb{L}^{2}(\mathbb{R}), \widehat{k} \in \mathbb{L}^{2}\left(I_{m}\right)$ y $\langle f, g\rangle_{I_{m}}=\int_{I_{m}} f(x) \overline{g(x)} d x$.

Veamos, como $S$ es el operador frame para $\left\{E_{a_{n}} T_{b_{m}} \widehat{g}\right\}$, tenemos

$$
S \widehat{h}=\sum_{m, n \in \mathbb{Z}}\left\langle\widehat{h}, E_{a_{n}} T_{b_{m}} \widehat{g}\right\rangle E_{a_{n}} T_{b_{m}} \widehat{g}=\sum_{m \in \mathbb{Z}} T_{b_{m}} \widehat{g}\left(\sum_{n \in \mathbb{Z}}\left\langle\widehat{h}, E_{a_{n}} T_{b_{m}} \widehat{g}\right\rangle E_{a_{n}}\right)
$$

pero,

$$
\begin{aligned}
\left\langle\widehat{h}, E_{a_{n}} T_{b_{m}} \widehat{g}\right\rangle & =\int_{-\infty}^{+\infty} \widehat{h} \overline{E_{a_{n}} T_{b_{m}} \widehat{g}(w)} d w=\int_{-\infty}^{+\infty} \widehat{h}(w) e^{-2 \pi i a_{n} w} \overline{\widehat{g}}\left(w-b_{m}\right) d w \\
\left\langle\widehat{h}, E_{a_{n}} T_{b_{m}} \widehat{g}\right\rangle & =\int_{I_{m}} \widehat{h}(w) \overline{\widehat{g}}\left(w-b_{m}\right) e^{\overline{2 \pi i a_{n} w}} d w \\
& =\left\langle\widehat{h} T_{b_{m}} \overline{\hat{g}}, E_{a_{n}}\right\rangle_{I_{m}}
\end{aligned}
$$

entonces

$$
S \widehat{h}=\sum_{m \in \mathbb{Z}} T_{b_{m}} \widehat{g}\left(\sum_{n \in \mathbb{Z}}\left\langle\widehat{h} T_{b_{m}} \overline{\hat{g}}, E_{a_{n}}\right\rangle_{I_{m}} E_{a_{n}}\right)
$$

tambien

$$
\begin{aligned}
\left\langle\widehat{h} T_{b_{m}} \overline{\widehat{g}}, E_{a_{n}}\right\rangle_{I_{m}} E_{a_{n}}(w) & \left.=\left(\int_{I_{m}} \widehat{h}(r) \overline{\widehat{g}\left(r-b_{m}\right.}\right) e^{-2 \pi i a_{n} r} d r\right) e^{2 \pi i a_{n} w} \\
& \left.=\left(\int_{I_{m}} \widehat{h}(r) \overline{\widehat{g}\left(r-b_{m}\right.}\right) e^{-2 \pi i a_{n}\left(r-b_{m}\right)} d r\right) e^{2 \pi i a_{n}\left(w-b_{m}\right)} \\
& =\left\langle\widehat{h} T_{b_{m}} \bar{g}, T_{b_{m}} E_{a_{n}}\right\rangle_{I_{m}} T_{b_{m}} E_{a_{n}}(w)
\end{aligned}
$$

por lo tanto

$$
\begin{aligned}
S \widehat{h} & =\sum_{m \in \mathbb{Z}} T_{b_{m}} \widehat{g}\left(\sum_{n \in \mathbb{Z}}\left\langle\widehat{h} T_{b_{m}} \overline{\hat{g}}, T_{b_{m}} E_{a_{n}}\right\rangle_{I_{m}} T_{b_{m}} E_{a_{n}}\right) \\
S \widehat{h} & =\sum_{m \in \mathbb{Z}} T_{b_{m}} \widehat{g}\left(S_{m}\left(\widehat{h} T_{b_{m}} \overline{\hat{g}}\right)\right) ; \widehat{h} \in \mathbb{L}^{2}(\mathbb{R}), \widehat{h} T_{b_{m}} \overline{\hat{g}} \in \mathbb{L}^{2}\left(I_{m}\right) .
\end{aligned}
$$




\section{Corolario 3.4.}

Con las hipótesis del teorema 3.2 , si $a_{n}=n a$ y $a=\frac{1}{2 \sigma}$ entonces

$$
S^{-1} \widehat{f}=\frac{1}{2 \sigma} \frac{\widehat{f}}{G} \text {. }
$$

Además

$$
S^{-1}\left(E_{n a} T_{b_{m}} \widehat{g}\right)=\frac{1}{2 \sigma} \frac{E_{n a} T_{b_{m}} \widehat{g}}{G}
$$

y

$$
E_{n a} T_{b_{m}} S^{-1} \widehat{g}=\frac{1}{2 \sigma} \frac{E_{n a} T_{b_{m}} \widehat{g}}{T_{b_{m}} G}
$$

\section{Demostración .}

En efecto, para $a_{n}=$ na y $a=\frac{1}{2 \sigma},\left\{\left(\frac{1}{\sqrt{2 \sigma}}\right) E_{n a}(x)\right\}$ es una base ortonormal, por consiguiente, $\left\{\left(\frac{1}{\sqrt{2 \sigma}}\right) E_{n a}(x)\right\}$ es un frame, para $\mathbb{L}^{2}([-\sigma, \sigma])$. Entonces ver teorema $3.2 ;\left\{E_{n a} T_{b_{m}} \widehat{g}\right\}$ es un frame para $\mathbb{L}^{2}(\mathbb{R})$ y $\widehat{f} T_{b_{m}} \overline{\widehat{g}} \in \mathbb{L}^{2}\left(I_{m}\right)$.

De este modo, se tiene que :

$$
\begin{aligned}
S \widehat{f} & =\sum_{m, n \in \mathbb{Z}}\left\langle\widehat{f}, E_{n a} T_{b_{m}} \widehat{g}\right\rangle E_{n a} T_{b_{m}} \widehat{g} \\
& =\sum_{m \in \mathbb{Z}} T_{b_{m}} \widehat{g}\left(\sum_{n \in \mathbb{Z}}\left\langle\widehat{f}, E_{n a} T_{b_{m}} \widehat{g}\right\rangle E_{n a}\right) \\
& =\sum_{m \in \mathbb{Z}} T_{b_{m}} \widehat{g}\left(\sum_{n \in \mathbb{Z}}\left\langle\widehat{f} T_{b_{m}} \overline{\hat{g}}, E_{n a}\right\rangle E_{n a}\right) \\
& =\sum_{m \in \mathbb{Z}} T_{b_{m}} \widehat{g}\left(2 \sigma \widehat{f} T_{b_{m}} \overline{\hat{g}}\right)=2 \sigma \widehat{f}\left(\sum_{m \in \mathbb{Z}}\left(T_{b_{m}} \widehat{g}\right)\left(T_{b_{m}} \overline{\hat{g}}\right)\right) \\
& =2 \sigma \widehat{f}\left(\sum_{m \in \mathbb{Z}}\left|\widehat{g}\left(w-b_{m}\right)\right|^{2}\right)=2 \sigma \widehat{f} G,
\end{aligned}
$$

esto es

$$
S \widehat{f}=2 \sigma \widehat{f} G
$$

Ahora, reemplazando $\widehat{f}$ por $S^{-1} \widehat{f}$ en $(11)$, tenemos

$$
\begin{aligned}
S\left(S^{-1} \widehat{f}\right) & =2 \sigma\left(S^{-1} \widehat{f}\right) G \\
\widehat{f} & =2 \sigma G\left(S^{-1} \widehat{f}\right)
\end{aligned}
$$

por lo tanto

$$
S^{-1} \widehat{f}=\frac{\widehat{f}}{2 \sigma G}
$$

Asimismo, reemplazando $\widehat{f}$ por $E_{n a} T_{b_{m}} \widehat{g}$ en $S^{-1} \widehat{f}=\frac{\widehat{f}}{2 \sigma G}$, se obtiene

$$
S^{-1}\left(E_{n a} T_{b_{m}} \widehat{g}\right)=\frac{E_{n a} T_{b_{m}} \widehat{g}}{2 \sigma G},
$$


observar que $E_{n a} T_{b_{m}} \widehat{g} \in \mathbb{L}^{2}(\mathbb{R})$.

Finalmente, como $2 \sigma G S^{-1} \widehat{g}=\widehat{g}$, entonces

$$
\begin{aligned}
E_{n a} T_{b_{m}}\left(G S^{-1} \widehat{g}\right) & =\frac{E_{n a} T_{b_{m}} \widehat{g}}{2 \sigma} \\
E_{n a} T_{b_{m}}\left(G S^{-1} \widehat{g}\right) & =\left(E_{n a} T_{b_{m}} S^{-1} \widehat{g}\right)\left(T_{b_{m}} G\right)=\frac{E_{n a} T_{b_{m}} \widehat{g}}{2 \sigma}
\end{aligned}
$$

por lo tanto

$$
E_{n a} T_{b_{m}} S^{-1} \widehat{g}=\frac{E_{n a} T_{b_{m}} \widehat{g}}{2 \sigma T_{b_{m}} G}
$$

Teorema 3.5. Sea $\sigma>0, y\left\{a_{n}\right\}$ una sucesión de números reales tal que $\left\{E_{a_{n}}\right\}$ es un frame para $\mathbb{L}^{2}([-\sigma, \sigma])$ con el operador frame $S_{0}$ y sea $\widehat{g}(w)=\frac{1}{\sqrt{2 \sigma}} \chi_{[-\sigma, \sigma]}(w)$. Entonces para toda $f$ función $\sigma$ - banda limitada, se tiene que

$$
f=\sqrt{2 \sigma} \sum_{n \in \mathbb{Z}} f\left(a_{n}\right) \phi_{n}
$$

donde $\phi_{n}(t)=\left(K_{n}(w)\right)^{\vee}, K_{n}=\sum_{m \in \mathbb{Z}}\left\langle E_{a_{n}} \widehat{g}, h_{m}\right\rangle_{I_{0}} h_{m}, h_{m}=S_{0}^{-1}\left(E_{a_{m}}\right), I_{0}=[-\sigma, \sigma]$.

\section{Demostración.}

Sea $b_{m}=2 \sigma m$.

Afirmación: $G(w)=\sum_{m \in \mathbb{Z}}|\widehat{g}(w-2 \sigma m)|^{2}=\frac{1}{2 \sigma}$

En efecto,

$$
\begin{aligned}
& \widehat{g}(w)=\frac{1}{\sqrt{2 \sigma}} \chi_{[-\sigma, \sigma]}(w)=\left\{\begin{array}{cc}
\frac{1}{\sqrt{2 \sigma}} ;-\sigma \leq w \leq \sigma \\
0 ; w \notin[-\sigma, \sigma]
\end{array}\right. \\
& \widehat{g}(w-2 \sigma m)=\frac{1}{\sqrt{2 \sigma}} \chi_{[-\sigma, \sigma]}(w-2 \sigma m)=\left\{\begin{array}{cl}
\frac{1}{\sqrt{2 \sigma}} & ;-\sigma \leq w-2 \sigma m \leq \sigma \\
0 & ; \text { en otro caso }
\end{array}\right. \\
& \widehat{g}(w-2 \sigma m)=\left\{\begin{array}{cl}
\frac{1}{\sqrt{2 \sigma}} & ; \sigma(2 m-1) \leq w \leq \sigma(2 m+1) \\
0 & ; \quad \text { en otro caso }
\end{array}\right. \\
& G(w)=\sum_{m \in \mathbb{Z}}|\widehat{g}(w-2 \sigma m)|^{2} \\
& =\ldots+|\widehat{g}(w+4 \sigma)|^{2}+|\widehat{g}(w+2 \sigma)|^{2}+|\widehat{g}(w)|^{2}+ \\
& +|\widehat{g}(w-2 \sigma)|^{2}+|\widehat{g}(w-4 \sigma)|^{2}+\ldots
\end{aligned}
$$

Para $m=-1 \Rightarrow-3 \sigma \leq w \leq-\sigma \Rightarrow \widehat{g}(w+2 \sigma)=0 \Rightarrow|\widehat{g}(w+2 \sigma)|^{2}=0$

Para $m=-2 \Rightarrow-5 \sigma \leq w \leq-3 \sigma \Rightarrow \widehat{g}(w+4 \sigma)=0 \Rightarrow|\widehat{g}(w+4 \sigma)|^{2}=0$

Para $m=0 \Rightarrow-\sigma \leq w \leq \sigma \Rightarrow \widehat{g}(w)=\frac{1}{\sqrt{2 \sigma}} \Rightarrow|\widehat{g}(w)|^{2}=\frac{1}{2 \sigma}$

Para $m=1 \Rightarrow \sigma \leq w \leq 3 \sigma \Rightarrow \widehat{g}(w-2 \sigma)=0 \Rightarrow|\widehat{g}(w-2 \sigma)|^{2}=0$

Para $m=2 \Rightarrow 3 \sigma \leq w \leq 5 \sigma \Rightarrow \widehat{g}(w-4 \sigma)=0 \Rightarrow|\widehat{g}(w-4 \sigma)|^{2}=0$ 
Por lo tanto $G(w)=\sum_{m \in \mathbb{Z}}|\widehat{g}(w-2 \sigma m)|^{2}=\frac{1}{2 \sigma}$.

Entonces por el teorema $3.2\left\{E_{a_{n}} T_{b_{m}} \widehat{g}\right\}$ es un frame para $\mathbb{L}^{2}(\mathbb{R})$. Además, el operador frame para $\left\{E_{a_{n}} T_{b_{m}} \widehat{g}\right\}$ es denotado por $S$, de esta forma, para todo $\widehat{f} \in \mathbb{L}^{2}(\mathbb{R})$, se tiene que

$$
\widehat{f}=\sum_{m, n \in \mathbb{Z}}\left\langle\widehat{f}, E_{a_{n}} T_{b_{m}} \widehat{g}\right\rangle S^{-1}\left(E_{a_{n}} T_{b_{m}} \widehat{g}\right)
$$

$\mathrm{y}$

$$
S \widehat{f}=\sum_{m, n \in \mathbb{Z}}\left\langle\widehat{f}, E_{a_{n}} T_{b_{m}} \widehat{g}\right\rangle S S^{-1}\left(E_{a_{n}} T_{b_{m}} \widehat{g}\right)=\sum_{m, n \in \mathbb{Z}}\left\langle\widehat{f}, E_{a_{n}} T_{b_{m}} \widehat{g}\right\rangle E_{a_{n}} T_{b_{m}} \widehat{g}
$$

Afirmación: $\quad\left\langle\widehat{f}, E_{a_{n}} T_{b_{m}} \widehat{g}\right\rangle=\left\{\begin{array}{c}\frac{1}{\sqrt{2 \sigma}} f\left(a_{n}\right) ; m=0 \\ 0 \quad ; m \neq 0\end{array}\right.$

En efecto,

$$
\begin{aligned}
\left\langle\widehat{f}, E_{a_{n}} T_{b_{m}} \widehat{g}\right\rangle & =\int \widehat{f}(w) \overline{E_{a_{n}} T_{b_{m}} \widehat{g}}(w) d w \\
& =\int \widehat{f}(w) e^{-2 \pi i a_{n} w} \overline{\widehat{g}(w-2 m \sigma)} d w
\end{aligned}
$$

pero, $\widehat{g}(w-2 m \sigma)=0, m \neq 0$ y como $\widehat{g}(w-2 m \sigma)=\frac{1}{\sqrt{2 \sigma}}$ para $m=0$, y $f$ es una función banda-limitada en $[-\sigma, \sigma]$, entonces

$$
\begin{aligned}
\left\langle\widehat{f}, E_{a_{n}} T_{b_{m}} \widehat{g}\right\rangle & =\int_{-\sigma}^{\sigma} \widehat{f}(w) e^{-2 \pi i a_{n} w} \frac{1}{\sqrt{2 \sigma}} d w \\
& =\frac{1}{\sqrt{2 \sigma}} \int_{-\sigma}^{\sigma} \widehat{f}(w) e^{-2 \pi i a_{n} w} d w \\
& =\frac{1}{\sqrt{2 \sigma}} f\left(a_{n}\right) \quad ; m=0
\end{aligned}
$$

Así

$$
\left\langle\widehat{f}, E_{a_{n}} T_{b_{m}} \widehat{g}\right\rangle=\left\{\begin{array}{r}
\frac{1}{\sqrt{2 \sigma}} f\left(a_{n}\right) ; m=0 \\
0 ; \quad m \neq 0
\end{array}\right.
$$

por lo tanto

$$
\widehat{f}=\sum_{n \in \mathbb{Z}} \frac{1}{\sqrt{2 \sigma}} f\left(a_{n}\right) S^{-1}\left(E_{a_{n}} T_{b_{m}} \widehat{g}\right)=\frac{1}{\sqrt{2 \sigma}} \sum_{n \in \mathbb{Z}} f\left(a_{n}\right) S^{-1}\left(E_{a_{n}} \widehat{g}\right)
$$

debido a que $T_{b_{m}} \widehat{g}(w)=\widehat{g}\left(w-b_{m}\right)=\widehat{g}(w-2 m \sigma)=\widehat{g}(w)$, si $m=0$. También

$$
S \widehat{f}=\frac{1}{\sqrt{2 \sigma}} \sum_{n \in \mathbb{Z}} f\left(a_{n}\right) E_{a_{n}} \widehat{g}=\frac{1}{\sqrt{2 \sigma}} \sum_{n \in \mathbb{Z}}\left\langle\widehat{f}, E_{a_{n}}\right\rangle_{I_{0}} E_{a_{n}} \widehat{g} .
$$

Además, como el operador frame del frame $\left\{E_{a_{n}}\right\}$ es $S_{0}$.

entonces

y

$$
S_{0}^{-1} \widehat{f}=\sum_{n \in \mathbb{Z}}\left\langle\widehat{f}, S_{0}^{-1} E_{a_{n}}\right\rangle_{I_{0}} S_{0}^{-1} E_{a_{n}}=\sum_{n \in \mathbb{Z}}\left\langle\widehat{f}, h_{n}\right\rangle_{I_{0}} h_{n}
$$

$$
S_{0} \widehat{f}=\sum_{n \in \mathbb{Z}}\left\langle\widehat{f}, E_{a_{n}}\right\rangle_{I_{0}} E_{a_{n}}
$$


Afirmación: $S \widehat{f}=\frac{1}{2 \sigma} S_{0} \widehat{f}$.

En efecto, de (12), tenemos $S \widehat{f}=\frac{1}{\sqrt{2 \sigma}} \sum_{n \in \mathbb{Z}}\left\langle\widehat{f}, E_{a_{n}}\right\rangle_{I_{0}} E_{a_{n}} \widehat{g}$, y como

$$
\widehat{g}(w)=\left\{\begin{array}{c}
\frac{1}{\sqrt{2 \sigma}} ;-w \leq \sigma \leq w \\
0 ; \text { en otro caso }
\end{array}\right.
$$

entonces

$$
\begin{aligned}
S \widehat{f} & =\frac{1}{\sqrt{2 \sigma}} \sum_{n \in \mathbb{Z}}\left\langle\widehat{f}, E_{a_{n}}\right\rangle_{I_{0}} E_{a_{n}}\left(\frac{1}{\sqrt{2 \sigma}}\right)=\frac{1}{\sqrt{2 \sigma}} \frac{1}{\sqrt{2 \sigma}} \sum_{n \in \mathbb{Z}}\left\langle\widehat{f}, E_{a_{n}}\right\rangle_{I_{0}} E_{a_{n}} \\
S \widehat{f} & =\frac{1}{2 \sigma} S_{0} \widehat{f} .
\end{aligned}
$$

debido a (14).

De este modo,

$$
S^{-1} \widehat{f}=(2 \sigma) S_{0}^{-1}(\widehat{f}) \text { en } \mathbb{L}^{2}([-\sigma, \sigma])
$$

En efecto, tomando $S^{-1} \widehat{f}$ por $\widehat{f}$ en la afirmación, obtenemos :

$$
\begin{aligned}
S\left(S^{-1} \widehat{f}\right) & =\frac{1}{2 \sigma} S_{0}\left(S^{-1} \widehat{f}\right) \\
\widehat{f} & =\frac{1}{2 \sigma} S_{0}\left(S^{-1} \widehat{f}\right) \\
S_{0}^{-1} \widehat{f} & =\frac{1}{2 \sigma} S_{0}^{-1}\left(S_{0}\left(S^{-1} \widehat{f}\right)\right)=\frac{1}{2 \sigma} S_{0}^{-1} S_{0}\left(S^{-1} \widehat{f}\right)=\frac{1}{2 \sigma} S^{-1} \widehat{f} .
\end{aligned}
$$

Por lo tanto

$$
S^{-1} \widehat{f}=(2 \sigma) S_{0}^{-1}(\widehat{f}) \text { en } \mathbb{L}^{2}([-\sigma, \sigma]) .
$$

Asi, de (13) y (15) obtenemos

$$
S^{-1}\left(E_{a_{n}} \widehat{g}\right)=(2 \sigma) S_{0}^{-1}\left(E_{a_{n}} \widehat{g}\right)=2 \sigma \sum_{m \in \mathbb{Z}}\left\langle E_{a_{n}} \widehat{g}, h_{m}\right\rangle_{I_{0}} h_{m}
$$

y reemplazando en $\widehat{f}=\frac{1}{\sqrt{2 \sigma}} \sum_{n \in \mathbb{Z}} f\left(a_{n}\right) S^{-1}\left(E_{a_{n}} \widehat{g}\right)$, obtenemos

$$
\begin{aligned}
\widehat{f} & =\frac{1}{\sqrt{2 \sigma}} \sum_{n \in \mathbb{Z}} f\left(a_{n}\right)\left((2 \sigma) \sum_{m \in \mathbb{Z}}\left\langle E_{a_{n}} \widehat{g}, h_{m}\right\rangle_{I_{0}} h_{m}\right) \\
\widehat{f} & =\frac{(2 \sigma)}{\sqrt{2 \sigma}} \sum_{n \in \mathbb{Z}} f\left(a_{n}\right)\left(\sum_{m \in \mathbb{Z}}\left\langle E_{a_{n}} \widehat{g}, h_{m}\right\rangle_{I_{0}} h_{m}\right) \\
\widehat{f} & =\sqrt{2 \sigma} \sum_{n \in \mathbb{Z}} f\left(a_{n}\right) K_{n},
\end{aligned}
$$

donde $K_{n}=\sum_{m \in \mathbb{Z}}\left\langle E_{a_{n}} \widehat{g}, h_{m}\right\rangle_{I_{0}} h_{m}$, y tomando la transformada inversa de Fourier, obtenemos

$$
(\widehat{f})^{\vee}=\sqrt{2 \sigma} \sum_{n \in \mathbb{Z}} f\left(a_{n}\right) \stackrel{\vee}{K}
$$

donde $\stackrel{\vee}{K}_{n}=\phi_{m}, h_{m}=S_{0}^{-1}\left(E_{a_{m}}\right)$ y $I_{0}=[-\sigma, \sigma]$. 
Por lo tanto

$$
f=\sqrt{2 \sigma} \sum_{n \in \mathbb{Z}} f\left(a_{n}\right) \phi_{n}
$$

Finalmente, si $\left\{E_{a_{n}}(w)\right\}$ es un frame exacto, entonces por la observación $2.9, S_{0}^{-1}\left(E_{a_{n}}\right)=h_{n}$, es la única sucesión biortonormal asociada con $\left\{E_{a_{n}}(w)\right\}$, esto es,

$$
\begin{aligned}
\left\langle E_{a_{n}}, S_{0}^{-1}\left(E_{a_{m}}\right)\right\rangle & =\delta_{m, n}, \mathrm{y} \\
K_{n} & =\sum_{m \in \mathbb{Z}}\left\langle E_{a_{n}} \widehat{g}, S_{0}^{-1}\left(E_{a_{m}}\right)\right\rangle_{I_{0}} S_{0}^{-1}\left(E_{a_{m}}\right) \\
& =\sum_{m \in \mathbb{Z}}\left\langle E_{a_{n}}\left(\frac{1}{\sqrt{2 \sigma}} \chi_{[-\sigma, \sigma]}\right), S_{0}^{-1}\left(E_{a_{m}}\right)\right\rangle S_{0}^{-1}\left(E_{a_{m}}\right) \\
& =\frac{1}{\sqrt{2 \sigma}} \sum_{m \in \mathbb{Z}}\left\langle E_{a_{n}}, S_{0}^{-1}\left(E_{a_{m}}\right)\right\rangle S_{0}^{-1}\left(E_{a_{m}}\right) \\
& =\frac{1}{\sqrt{2 \sigma}} S_{0}^{-1}\left(E_{a_{n}}\right)=\frac{1}{\sqrt{2 \sigma}} h_{n}
\end{aligned}
$$

Por lo tanto

$$
K_{n}=\frac{1}{\sqrt{2 \sigma}} h_{n}
$$

y

$$
\begin{aligned}
\phi_{n}(t) & =\left(K_{n}(w)\right)^{\vee}=\int K_{n}(w) e^{-2 \pi i t w} d w=\int \frac{1}{\sqrt{2 \sigma}} h_{n}(w) e^{-2 \pi i t w} d w \\
& =\frac{1}{\sqrt{2 \sigma}} \int h_{n}(w) e^{-2 \pi i t w} d w=\frac{1}{\sqrt{2 \sigma}} \psi_{n}(t)
\end{aligned}
$$

\section{Conclusiones}

1.- En particular, si $\left\{E_{a_{n}}(w)\right\}$, es un frame exacto, entonces $f=\sum_{n \in \mathbb{Z}} f\left(a_{n}\right) \psi_{n}$, donde $\psi_{n}(t)=\int_{-\sigma}^{\sigma} h_{n}(w) e^{-2 \pi i t w} d w$.

2. La técnica de los frames y duales frames en $\mathbb{L}^{2}(\mathbb{R})$ son usados para generar resultados de muestreo como la asociada en el teorema 3.5.

3. Finalmente, la técnica de los frames es mucho mas refinada que la técnica de la dualidad entre una base y su base biortogonal. 


\section{REFERENCIAS BIBLIOGRÁFICAS}

[1] BEYLKIN G., COIFMAN R., ROKHLIN V. Fast Wavelet Transforms and numerical algorithms. Comm. Pure and Appl. Math., 44, (1991), pp.141-183.

[2] BURKE-HUBBAR, B. Ondes et Ondelettes, pour la science, París, (1995).

[3] CHUI, C., An Introduction to Wavelets. Academic Press, Boston, (1992).

[4] CHUI, C., Wavelets: a Tutorial in Theory and Applications. Academic Press, Boston, (1992).

[5] COHEN A., DAUBECHIES I., \& P. VIAL. Wavelets on the interval and fast wavelet transform. Journal of Applied and Computational Harmonic Analysis 1, (1993), pp. 54-81.

[6] DAUBECHIES I., Ten lectures on Wavelets, CBMS-NSF Regional Conference Series in Applied Mathematics, SIAM Publ. Philadelphia, (1992).

[7] DAUBECHIES I. Orthonormal bases of Compactly Supported Wavelets. Comm. Pure and Appl. Math. 41, (1988), pp. 909-996.

[8] LOKENATH D., Wavelets transforms and their Applications. Birkhäuser, Boston, (2002). 